\title{
On the Relation Between the Red Blood Cells and the Antibody Formation
}

\author{
By \\ Sakae Masuda \\ Department of Anatomy, Faculty of medicine, Juntendo University \\ Hongo, Tokyo
}

(Comm. by T. F U R U H A T A, M. J. A., Dec. 13, 1956)

The site of the formation of antibodies is not yet definite inspite of many studies. The reticuloendothelial cells, lymphocytes (modified later by $\mathrm{Ehrlich}$ and others) and plasma cells have been suggested as the place of the formation of antibodies.

Furthermore, concerning the process of the antibody formation only hypotheses have been proposed.

During the studies of the structure of the red cells, I have found that the latent period before the antibody production corresponds to the important period during which the antibody matures in the red blood cells.

Furthermore, I have proved histologically and serologically that when it disappears, the antibody appears in the serum. This compelled me to reconsider the process of the formation of antibodies.

\section{Materials and Methods}

Macerated liver, kidney and lung were injected as antigens into 30 rabbits. The blood was taken daily from them just before the injection and stained by M-A and Giemsa methods. The quantities of antigens and antibodies in the red cells and the serum were measured by serological method.

In order to completely isolate the red cells and the serum, after the red cells were washed three times with the physiological saline and separated by centrifugation, they were hemolyzed, adjusted for $\mathrm{pH}$, diluted to various concentrations and their \pm points were determined by the turbidity reaction with antigens. The serum which has shown negative turbidity reaction was tested for the appearance of the antibody by precipitation and agglutination reactions. 


\section{Results}

While four days and seven days after the cessation of the antigen injection, the antibody is not found in the serum, the antige (antibody?) formation in the red cells is conspicuously increased in amount (Fig. 1. and 2).

Twelve days after the cessation of the antigen injection, the

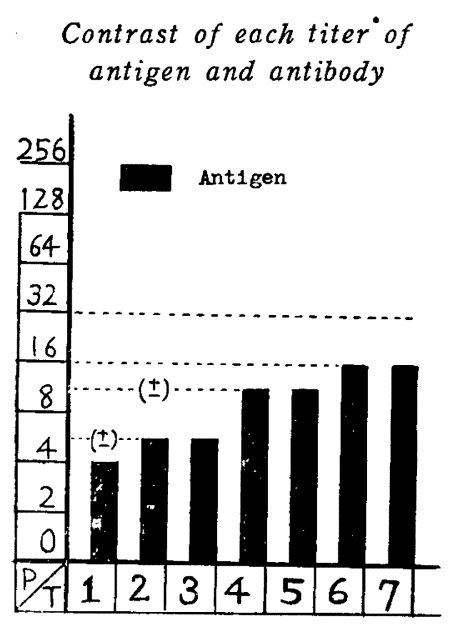

Fig. 1. 4 days after cessation of the antigen injection.

\section{Contrast of each titer of antigen and antibody}

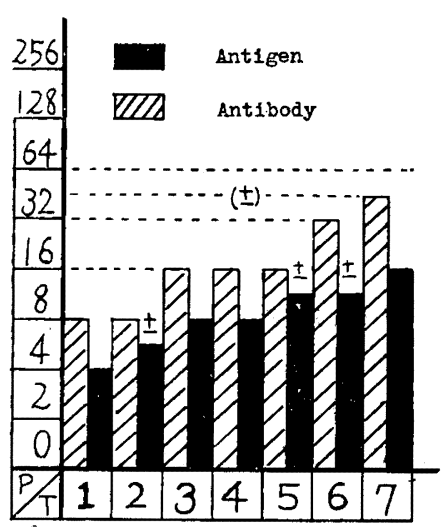

Fig. 3. 12 days after cessation of the antigen injection.
Contrast of each titer of antigen and antibody

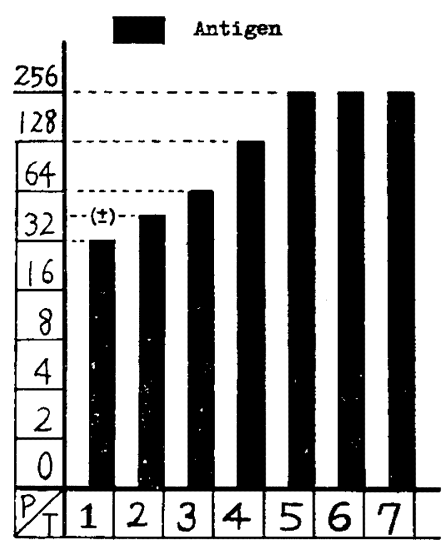

Fig. 2. 7 days after cessation of the antigen injection.

\section{Contrast of each titer of antigen and antibody}

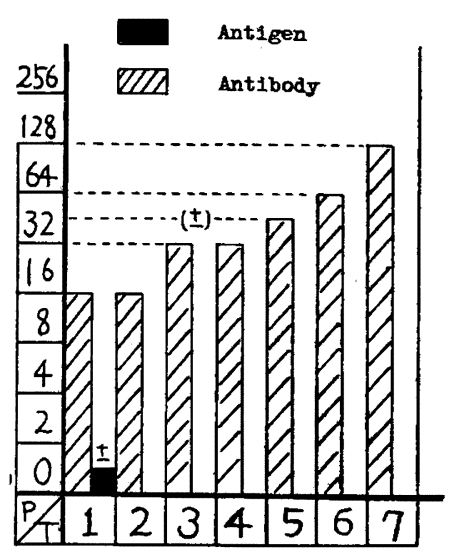

Fig. 4. 17 days after cessation of the antigen injection. 
amount of the antibody in the serum increases whereas the quantity of the antigen (antibody?) in the red cells decreases. Seventeen days after it, the antigen (antibody?) in the red cells disappears almost completely and the amount of the antibody in the serum increases conspicuously (Fig. 3. and 4.).

At this time in the Giemsa preparations of the blood smears, large polychromatic red cells are found to increase in number in correlation with the increase of the antigen in the red cells. They decrease in number as the antigen in the red cells decreases and disappear at about the time the antibody appears in the serum. Fig. 5.-7. show the percentage of these changed red cells. It indicates that after the cessation of the antigen injection, they increase and then decrease, and finally disappear after desensitization. Fig. 5.-7. show graphically the appearance of these red cells in percentage after the antigen injection and the days of their appearances. It is seen that when dog's liver is used as the antigen, eight days after the cessation of the injection, the largest number of these red cells appear. In the case of guinea-pig's liver and kidney used as antigens, the largest number of these red cells appear at the same time. They decrease as the antibody appears in the serum and then disappear.

The rise of the antibody titers in the serum is in a reverse

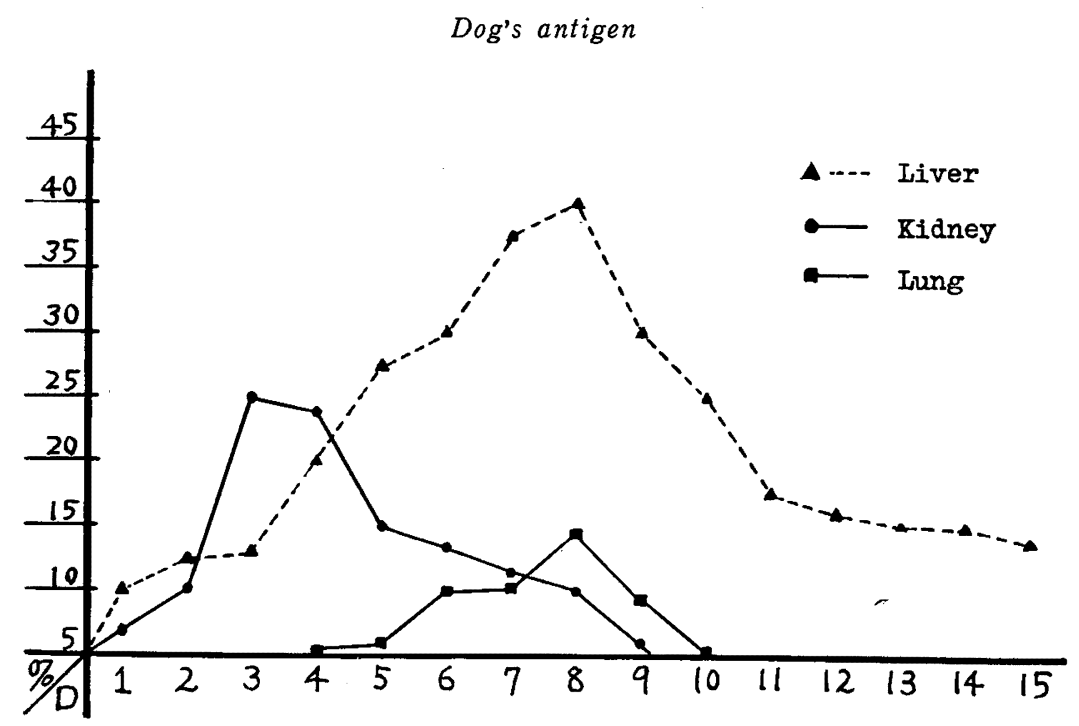

Fig. 5. Percentage of the changed red cells after cessation of the antigen injection. 
relation to the number of the changed red blood cells during the early period of the formation of the antibody. Red blood cells disappeared completely by desensitization temporarily reappear in a small number after desentization and then disappear completel This relation is clearly indicated in Fig. 5.-7. In short the forma-

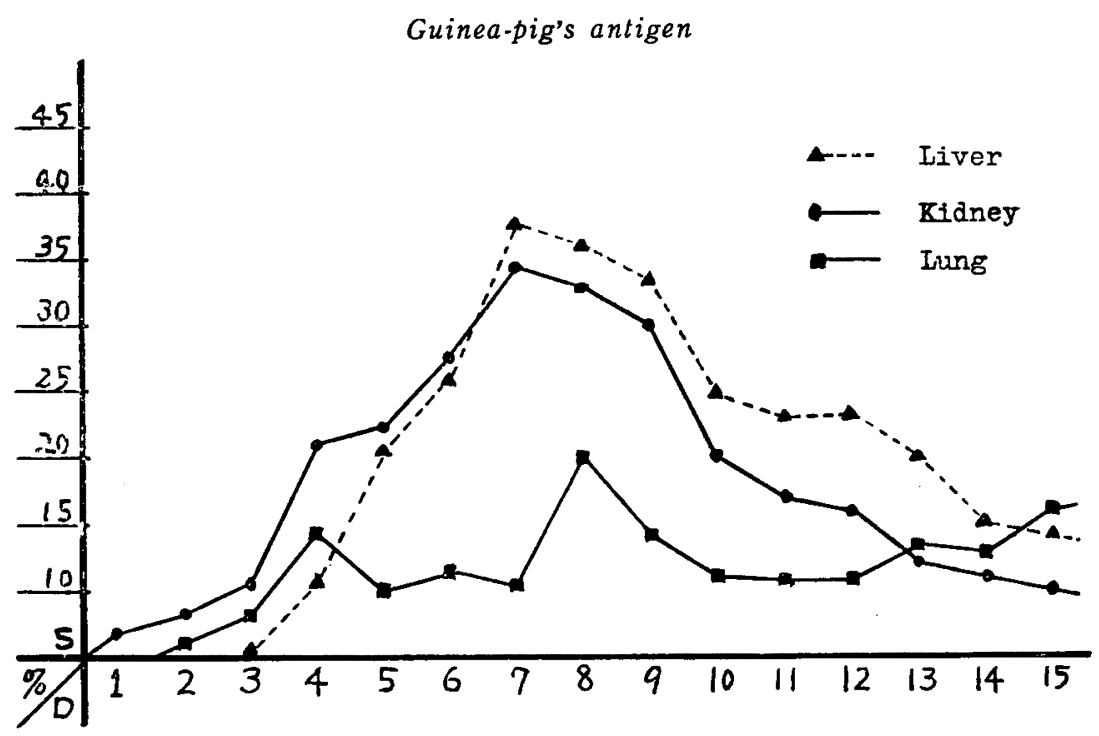

Fig. 6. Percentage of the changed red cells after cessation of the antigen injection.

Rat's antigen

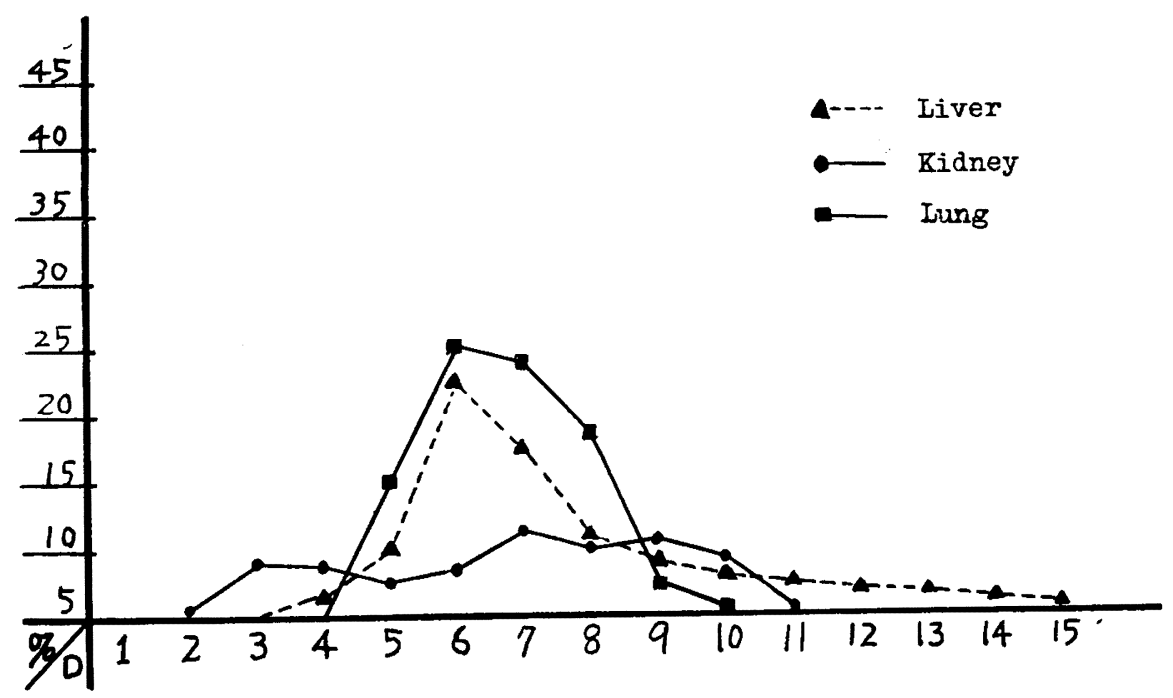

Fig. 7. Percentage of the changed red cells after cessation of the antigen injection. 
tion and the increase and decrease of the number of polychromatic large red cells are in parallel with the increase and decrease of the antigen (antibody?) of the red cells and they disappear when the antibody appears in the serum. At this time which is called the latent period in serology, the antigen entered into the red cells matures in them as the internal antigen (this may be called antibody) and the preparation is made for the antibody to appear in the serum. This seems to be proved by the appearance of the polychromatic red cells and the serological method.

\section{Discussion}

Only indirect methods have been used for the detection of the place where the antibody is formed. This seems to be the reason why the determination has not been made yet. A proof by direct histochemical methods has not yet been obtained, but I think that I have approached a step closer to this hope. I am studying now the changes of red cells after injections of the antigen by M-A method.

Since the lymphocyte theory has been modified, at present the reticuloendothelial system and the plasma cells are thought to be the place of the origin of antibodies. From the observations presented above I cannot but believe that the red blood cells produce antibodies.

Let us reconsider the experiential facts once more. Since the blocking of the reticuloendothelial systhem and splenectomy cause the disappearance and decrease of red cells, the formation of the antibody is naturally poor. The organs with concentrated antigens coincide with those with concentrated antibodies. These are, for instance, the bone marrow, spleen and liver. These organs contain a large number of red cells and are closely related to this, and the results of the tissue cultures are also in agreement with this in general.

Since the internal antigen found to exist in red cells is proved in the hemolyzed blood of the red cells by means of the method used to prove the antibody, this could be called the antibody. Although it is not known yet whether the antibody in the red cells and the antibody in the serum are the same thing, they are undoubtedly closely related. 


\section{Conclusion}

I cannot but think that the red blood cells are related to the formation of antibodies.

\section{References}

1) Masuda, S.: Proc. Japan Acad. Vol. 32, No. 8 644-647 (1956).

2) : Juntendo Med. J. Vol. 2, No. 3 131-143 (1956). 